\title{
Cardiac MRI and FDG-PET in the diagnosis of cardiac sarcoidosis
}

\author{
Richard Coulden ${ }^{*}$, Hefin Jones, Emer Sonnex, Indrajeet Das, Jonathan Abele \\ From 17th Annual SCMR Scientific Sessions \\ New Orleans, LA, USA. 16-19 January 2014
}

\section{Background}

Sarcoidosis is a multisystem disorder with cardiac involvement in $25 \%$ of cases [1]. Diagnosis of cardiac sarcoidosis is challenging with FDG-PET and cardiac MRI (CMRI) proving most reliable. We compare FDG-PET and CMRI with delayed enhancement (LGE) in patients with biopsy proven extra-cardiac sarcoidosis being investigated for cardiac involvement.

\section{Methods}

30 patients meeting Japanese Ministry of Health \& Welfare guidelines [2] for clinical cardiac sarcoidosis were investigated with FDG-PET CT (Gemini TF Philips) and CMRI (Aera 1.5T Siemens) on the same day. Patients undergoing FDG-PET followed a 24 hour low-carbohydrate diet and overnight fast [3]. CMRI examination included SSFP assessment of left ventricular (LV) func- tion, short axis T2-weighted STIR and PSIR-LGE 10 minutes post $0.2 \mathrm{mmol} / \mathrm{kg}$ GdDTPA. Images were reviewed by experienced readers blinded to the results of the other examination. FDG-PET was considered positive if any segment (AHA 17 segment model) had an SUVmax > 3.6 (3). CMRI was considered positive if any segment showed 'sarcoid-type' LGE. In no case was edema present on STIR imaging without LGE in the same segment on subsequent PSIR.

\section{Results}

FDG-PET and CMRI were positive in 10 patients; FDGPET + ve and CMRI -ve in 3; FDG-PET -ve and CMRI +ve in 4; both -ve in 13. Distributions of FDG and LGE throughout the myocardium are summarized in Figure 1. In 2 cases where FDG and CMRI were +ve, LGE was in an ischemic pattern and both patients had known
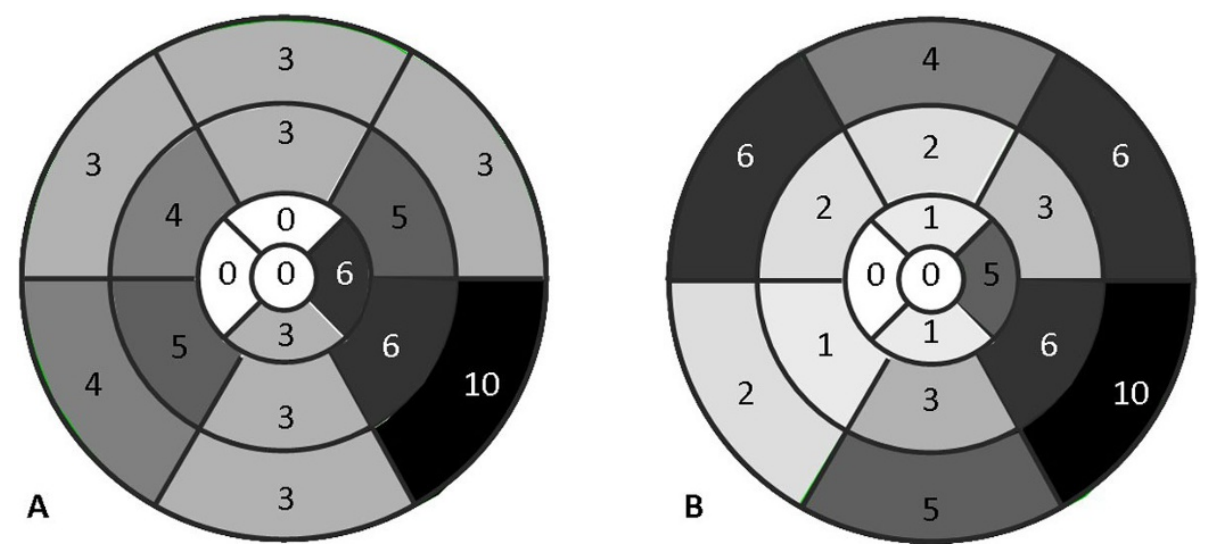

Figure 1 LV distribution of LGE (A) and significant myocardial FDG uptake (B) for all $\mathbf{3 0}$ patients. By both modalities, cardiac sarcoidosis predominantly involves the lateral wall with relative sparing of the distal anterior wall, distal septum and apex.

Dept Radiology \& Diagnostic Imaging, University of Alberta Hospital,

Edmonton, Alberta, Canada 
recent iscemic events (sub-endocardial). 6 of the remaining 8 had impaired LV function ( $\mathrm{EF}<50 \%$ ). All FDG +ve/CMRI -ve cases had intense mediastinal/lung FDG uptake. All 4 FDG -ve/CMRI +ve had either longstanding sarcoidosis ( $>2$ years) or absent node/lung FDG activity. Seven of the cases, -ve by both modalities, had no significant or minimal node/lung FDG uptake (SUVmax < 2.5).

\section{Conclusions}

Previous single modality studies have suggested sensitivity and specificity for FDG-PET of $89 \%$ and $78 \%$ and CMRI of $75 \%$ and $77 \%$ respectively. In our study, $43 \%$ showed no cardiac sarcoidosis by either modality and half of these had minimal or no FDG-PET evidence of active sarcoidosis elsewhere. Are these false -ve FDG-PET/ CMRI studies or are the clinical criteria too sensitive? Our findings suggest FDG-PET and CMRI-LGE show different degrees of cardiac sarcoid involvement: FDG-PET indicating active inflammatory disease, LGE showing severe edema and scar. Those patients with LGE and no myocardial FDG uptake appear to have 'burnt-out' disease.

\section{Funding}

None.

Published: 16 January 2014

\section{References}

1. Iwai: Sarcoidosis 1994.

2. Hiraga: Japanese Ministry of Health \& Welfare. 1993.

3. Coulden: Eur Radiol 2012.

doi:10.1186/1532-429X-16-S1-P299

Cite this article as: Coulden et al:: Cardiac MRI and FDG-PET in the diagnosis of cardiac sarcoidosis. Journal of Cardiovascular Magnetic

Resonance 2014 16(Suppl 1):P299.
Submit your next manuscript to BioMed Central and take full advantage of:

- Convenient online submission

- Thorough peer review

- No space constraints or color figure charges

- Immediate publication on acceptance

- Inclusion in PubMed, CAS, Scopus and Google Scholar

- Research which is freely available for redistribution

Submit your manuscript at www.biomedcentral.com/submit
C Biomed Central 